MS03.01.05 VRML AS A TOOL FOR EXPLORING COMPLEX STRUCTURES. A. Le Bail, Laboratoire des Fluorures, URA CNRS 449, Université du Maine, 72017 Le Mans, France.

Exploring a crystal structure from the inside and really understanding it: the Virtual Reality Modelling Language (1) allows this and much more. Simple applications can readily be produced without specialist knowledge. Use your favourite drawing program, decide what you would like to see (select atoms), convert your data into a standard. wrl file and then view the result on almost any platform. A PDB (Protein Data Bank) to VRML converter already exists (2). The whole operation (apart from PDB2VRML which is UNIX only) can even be carried out on just a fast PC although graphics workstations improve productivity. For inorganic structures an excellent procedure is to convert SHELX ins into pdb files using BABEL (3), select those atoms suitable for the display required, verify the result with RASMOL (4) and finally create automatically the wrl file with PDB2VRML.A change in the pre-defined radius and colors can be undertaken by editing the ASCII wnl file. A more direct builder is xtal-3d (5) for UNIX platforms, a PC adaptation is planned.

The most exciting moment during the course of a crystal structure determination occurs when enough atomic positions are known to be able to draw at least a partial model. The less exciting is when it is realized that the structure is so complex that it will be necessary to build a real 3D paper (or ball and stick) model in order to be sure that nothing has been overlooked in the structure description. VRML can help one to do almost the whole job in a few minutes starting from standard drawing files. Visual applications are limited only by ones own imagination. For the specialist it is feasible to create 3D crystallographic scenarios, calling objects stored at different URLs on the net. The real usefulness to crystallography of 3D mark-up is an open question.

1. J.C. Hardenbergh, http://www.oki.com/vrml/VRML_FAQ.html

2. H.Vollhardt, http://ws05.pc.chemie.th-darmstadt.de/vrml/ pdb2vrml.html

3. P. Walters \& M. Stahl, http:/mercury.aichem.arizona.edu/babel.html

4. R. Sayle, ftp://ttp.des.ed.ac.uk/pub/rasmol

5. M. Hewat, http://193.49.43.3/dif/3D_crystals.html

\section{Computing II}

General Advances \& Application

MS03.02.01 PHASE IMPROYEMENT AND EXTENSION IN PROTEIN CRYSTALLOGRAPHY. Giuseppe Zanotia, Dritan Siliquib and Carmelo Giacovazzob, aDipartimento di Chimica Organica e Centro Studi Biopolimeri, Via Marzolo 1, 35131 Padova, Italy, bDip. Geomineralogico, U. di Bari, Via Orabona, Bari, Italy.

In protein structure solution with the MIR technique, very often the isonorphism of heavy-atom derivatives does not extend beyond $3 \AA$ resolution or so. Usually, a set of phases obtained with the isomorphous replacement method must be improved further, in order to obtain an interpretable electron density map. In the single isomorphous replacement (SIR) approach, this is even a conditio sine qua non. Two methods aimed to overcome these problems will be discussed.

In the first, single isomorphous replacement techniques are integrated with direct nethods in order to solve the ambiguity of the phase.

The second consists of a procedure of phase extension and improvement, based on discrete Hilbert transforms? This proce- dure is based on a completely different principle from those previously described and has the advantage of being absolutely modelindependent. The method has been tested using simulated diffraction data of a small molecule and of a protein crystal. Starting from a randomly incomplete set of correct phases, it allows calculation of the unknown phases. Moreover, a set of phases affected by a mean phase error of $\pm 90^{\circ}$ can be improved to a mean error of $\pm 25^{\circ}$, if the correct figures of merit for the reflections are known. The performance and the limitations of the technique, as well as the perspectives for further developements, will be discussed.

References

1C. Giacovazzo, D. Siliqui, J.G. Platas, H.J. Hecht, G. Zanotti and B. York "The Crystal Structure Solution of Proteins by Direct Methods. VI. Complete Phasing up to Derivative Resolution" Acta Cryst. D. 1996, in press (and references therein).

2 G. Zanotti, F. Fogale and G. Capitani "The use of Discrete Hilbert Transforms in Phase Extension and Improvement", Acta Cryst., submitted

MS03.02.02 SOFTWARE FOR LATTICE IDENTIFICATION IN G-6. Herbert J. Bernstein, Bernstein+Sons, 5 Brewster Lane, Bellport, NY 11713-2803, USA and Lawrence C. Andrews, Thuridion S.E., 269 Mt. Hermon Rd., Scotts Valley, CA 95066 USA

We present the current state of software for Bravais lattice identification in G-6, based on a Fortran program which will accept experimental cell information on-line or from mmCIF or PDB format data files.

The coordinate system of $G-6$, defined by $(a, a),(b, b),(c, c)$, $2(b, c), 2(a, c), 2(b, c)$ is one in which a meaningful measure for quality of lattice identification is provided by Euclidean distances from appropriate linear subspaces. A modest combinatorial complexity is introduced by the need to evaluate multiple alternative distances not only from a given cell, but from nearby nearly-reduced cells. If the original reduced cell is not highly skewed the identification of a minimal distance requires only one to two shells of operations from the group of reduction transformations.

MS03.02.03 A LOCAL MACROMOLECULAR STRUCTURE DATABASE FOR CRYSTALLOGRAPHIC LABORATORIES. Philip E. Bourne and Ilya N. Shindyalov, San Diego Supercomputer Center, PO Box 85608, San Diego CA 921869784

As the number of macromolecular structures continues to grow exponentially, the need for a compact, easy to load and easy to query labortaory based database system would seem important. Such a database should be capable of loading all or a subset of the structures found in the Protein Data Bank (PDB) as well as maintaing local data which is in PDB format. The ideal system should contain native and derived data, should run on a variety of Unix platforms and should have a Web-based graphical interface to query the database. This paper reports on the design, capabilities and availability of such a database system. The San Diego Supercomputer Center (SDSC) version of the database is available via the World Wide Web on multiple servers (http://www.sdsc.edu/ moose) and is within 24 hours of being current with the PDB native distribution as found in the PDB ftp archives. Using similar compression algorithms as found in WPDB [1] reduces data strorage requirements 10 fold over native data without any loss of precision and aklso includes additional derived data. Apart from the obvious types of queries based on authors, protein names and other basic information, queries can be made with respect to characteristics of the polypepticle chain, for example, sequence patterns (with gaps), patterns of secondary structure elements, 
percentage of different kinds of secondary structure elements. The most extensive queries are those based on patterns of amino acid properties. It is possible to search for patterns combing such properties as environmental exposure, hydrophobicity, volume, polarity, isoelectric point, B values and so on. By taking properties from a given primary sequence and applying appropriate thresholds threading is possible. Altematively, the starting properties can be taken from a known structure and structure similarity determined. A range of graphical tools can be applied to structures found in querying. These include plots of various property patterns, contact maps and 3-D images. The database system can be obtained by contacting the first author.

[1] I.N Shindyalov and P.E.Bourne (1995), J. App. Cryst. 28(6) 847-852.

MS03.02.04 A UNIVERSL DATA EXCEANGE FORMAT FOR CRYSTALLOGRAPHY. B. MCMahon, International Union of Crystallography, 5 Abbey Square, Chester CH1 2HU, England

The Crystallographic Information File (CIF) (Hall, Allen \& Brown, 1991) is now the common exchange mechanism for crystallographic data supported by the IUCr. Increasingly, crystallographic program packages are able to read, write and validate CIF data. The file format has a simple syntactic structure, uses the ASCII character set for maximum portability between operating platforms, and is simply extensible. Dictionaries of recognised data names are constructed in a machine-readable formalism, allowing data relationships to be built and validated automatically.

This presentation will describe many current CIF applications, and discuss some of the considerations for successful programming of CIF software. There will also be a demonstration of how the IUCr uses standard software packages to check the quality of crystallographic data reported in its journals.

References

Hall, S. R., Allen, F. H. \& Brown, I. D. (1991). Acta Cryst. A47, 655-685.

MS03.02.05 SIRWARE by G. Cascarano(+), A. Altomare(t), C. Giacovazzo(+), A. Guagliardi(+), A.G.G. Moliterni(+), D. Siliqi (+), M.C. Burla(*), G. Polidori( ${ }^{(*)}$ \& M. Camalli( $)(+)$ Istituto di Ricerca per lo Sviluppo di Metodologie Cristallografiche - CNR c/o Dipartimento Geomineralogico - Campus Universitario Via E. Orabona, 4 - 70125 Bari - Italy. ${ }^{*}$ Dipartimento di Scienze della Terra, Universita, 06100 Perugia, Italy. (")CNR, Istituto di Strutturistica Chimica G. Giacomello, C.P.10,00016 Monterotondo Stazione (RM), Italy.

New releases of our software and new programs will be presented. The covered fields are:

- treatment of powder data

A new integrated software based on EXTRA and SIRPOW exploiting all the synergies between extraction intensities step and the Direct Methods application.

- single crystal

The new version of the Direct Methods program SIR ( SIR96)

including the treatment of electron diffraction data.

- protein data

A new program based on the integration of Direct Methods and isomorphous derivative techniques, solvent flattening and histogram matching.

A powerful and effective graphic interface is available in this software, suitable for all common platforms.

The programs will be free of charge for academic institutions.
MS03.02.06 COMPUTATIONAL ASPECTS OF XD. T. Richter, Institute for Crystallography, Free University of Berlin, Germany, T. Koritsanszky, S. Howard, P.R. Mallinson, Z. Su, N.K. Hansen

$\mathrm{XD}$ is a computer program package for multipole refinement and analysis of electron densities from diffraction data. It has been developed within an IUCr project by an iternational programing team. The main task of the package is to extract electronic properties from Bragg reflections and to perform topological analysis on 3D properties such as the electron density, its Laplacian function /1/ and the electrostatic potential. The package consists of the following programs:

-XDINI - convert data from conventional packages

-XDEDIT - edit master input file

-XDLSM - least squares program

-XDFOUR - general plane fourier program

-XDFFT - fast-fourier program

-XDPROP - electronic properties, topological analysis

-XDGEOM - geometrical analysis

-XDSTAT - statistical analysis

-XDVIB - thermal motion analysis

-XDGRAPH - graphical output

These programs will be described and their main computational aspects discussed.

/1/ R.F.W. Bader, Atoms in Molecules: A Quantum Theory, Oxford University Press (1990).

MS03.02.07 PARAMETERISING WEAK INTERACTIONS. S.J. Maginn, Molecular Simulations, 240/250 The Quorum, Barnwell Rd., Cambridge CB5 8RE, UK G.R. Desiraju, Dept. of Chemistry, University of Hyderabad, Hyderabad 500134, India

Forcefield parameters to account for a range of so-called "weak" interactions, such as C-H...O, have been successfully derived and tested.

Many crystal structures depend for their integrity on one or more of a range of so-called "weak" interactions. Examples of these include $\mathrm{C}-\mathrm{H} \ldots . . . \mathrm{O}, \mathrm{C}-\mathrm{H} . . . \mathrm{N}, \mathrm{N} . . . \mathrm{Cl}$ etc.

No currently widely available molecular mechanics forcefield contains parameters to account for these interactions. Consequently, this is a weakness of the emerging forcefield-based crystal structure prediction methodologies (e.g. those of Gavezzotti (l), and Karfunkel and Gdanitz (2)) - using such forcefields, structures containing "weak" interactions can never be accurately predicted.

A range of crystal structures containing $\mathrm{C}-\mathrm{H} \ldots . . \mathrm{O}$ and $\mathrm{C}-\mathrm{H}$..... interactions has been used, along with the tools in the Cerius2 molecular modelling suite (such as molecular mechanics minimisation, and the Karfunkel and Gdanitz structure prediction method) (3), to produce a customised forcefield which accounts for these, and which it is hoped will lead to more accurate structure predictions in future.

(1) A. Gavezzotti; J. Am. Chem. Soc. 113 (1991), p4622

(2) H.R. Karfunkel, R.J. Gdanitz; J. Comp. Chem. 13 (1992), p1171

(3) Cerius2, from Molecular Simulations Inc., 9685 Scranton Rd., San Diego, CA 92121-3752, USA, or 240/250 The Quorum, Barnwell Rd., Cambridge CB5 SRE, UK 